\title{
RESEARCH HIGHLIGHT COVID-19: towards understanding of pathogenesis
}

\author{
Wei Cao ${ }^{1}$ and Taisheng $\mathrm{Li}^{1}$ \\ Cell Research (2020) 30:367-369; https://doi.org/10.1038/s41422-020-0327-4
}

\begin{abstract}
Since the end of 2019, COVID-19 has caused considerable mortality and morbidity worldwide, and become a priority of the global society. Better understanding the pathogenesis of COVID-19 will provide important insight into its management.

The pandemic of coronavirus disease 2019 (COVID-19) is spreading rapidly. Although the cause was quickly identified as a new coronavirus named SARS-CoV-2, our knowledge of this novel virus remains very limited. High infectivity of the virus, lack of effective antivirals and vaccines, and potentially large asymptomatic populations, have made management of COVID19 extremely challenging. In addition to rapid medical responses, continuous efforts to better understand the pathogenesis of this disease will undoubtedly enlighten the optimal management of the growing pandemic.
\end{abstract}

SARS-CoV- 2 belongs to the $\beta$-coronavirus family, and is partially related with the known SARS-CoV ( 79\% similarity) and MERS-CoV ( $50 \%$ similarity) according to genome sequencing. ${ }^{1}$ Same as SARS-CoV, SARS-CoV-2 uses angiotensin-converting enzyme 2 (ACE2) as its main receptor, which is broadly expressed in vascular endothelium, respiratory epithelium, alveolar monocytes, and macrophages. ${ }^{1}$ The main transmission route is through direct or indirect respiratory tract exposure. Of note, SARS-CoV-2 is capable of active replication in the upper respiratory tissues, ${ }^{1,2}$ as demonstrated by successful live virus isolation from throat swabs and detection of viral subgenomic messenger RNA (sgRNA) in cells of upper respiratory tract. ${ }^{2}$ Tropism of the upper respiratory tissue probably explains continuous pharyngeal shedding of the virus and a more efficient transmission of SARS-CoV-2 than SARS-CoV when symptoms are still minimal and restricted to the upper respiratory tract. Later in the disease course, COVID-19 resembles SARS in terms of viral replication in the lower respiratory tract, and generates secondary viremia, followed by extensive attack against target organs that express ACE2, such as heart, kidney, gastrointestinal tract and vast distal vasculature. This process of viral spreading correlates with the clinical deterioration, mainly taking place around the second week following disease onset. However, it has been generally recognized that disease exaggeration till the late stage is not only attributed to direct viral damage, but also a consequence of immune-mediated injury induced by SARS-CoV- 2 . Of note, two distinctive features have been noticed in severe and critical patients with COVID-19, progressive increase of inflammation and an unusual trend of hypercoagulation.

Although the concept of inflammatory storm remains controversial, there is no doubt that immune-mediated inflammation plays an important role in the pathogenesis of COVID-19, just as it did in SARS. The progression of COVID-19 was associated with a continuous decrease in lymphocyte count and significant elevation of neutrophils. Meanwhile, inflammatory markers were markedly elevated including C-reactive protein, ferritin, interleukin
(IL)-6, IP-10, MCP1, MIP1A, and TNFa. Reduced lymphocyte count and elevated levels of ferritin, IL-6 and D-dimer were reported in various studies to be associated with increased mortality of COVID-19. ${ }^{3,4}$ Mechanisms underlying the progressive lymphopenia in severe and critical COVID-19 patients remain unclear. Subset analysis showed a general decrease in B cells, T cells, and natural killer (NK) cells, which was more prominent in severe cases. ${ }^{3} \mathrm{Xu}$ et al. ${ }^{5}$ reported increased level of $\mathrm{CD}^{+}{ }^{+} \mathrm{T}$-cell activation (measured by proportions of CD38 and HLA-DR expression) despite the reduction in $\mathrm{CD}^{+}{ }^{+}$-cell count in one critically ill COVID-19 patient. Lymphopenia was also an important feature of SARS patients, and decline of both $\mathrm{CD}^{+}{ }^{+}$and $\mathrm{CD} 8^{+} \mathrm{T}$ lymphocytes often preceded the radiographic changes. ${ }^{6}$ Although direct infection of macrophages and lymphocytes by SARS-CoV was indicated by one study, ${ }^{7}$ rapid reduction of lymphocyte counts in SARS was further attributed to two mechanisms, redistribution of the circulating lymphocytes or depletion of lymphocytes through apoptosis or pyroptosis. ${ }^{6,8}$

Currently, no viral gene expression has been observed in peripheral blood mononuclear cells (PBMCs) of patients with COVID-19. ${ }^{9}$ However, Wang et al. ${ }^{10}$ indicated that T lymphocytes may be more permissive to SARS-CoV-2 than to SARS-CoV, possibly through an endocytosis pathway triggered by the spike protein. As ACE2 is not readily expressed on lymphocytes, the efficiency of membrane fusion and to what extent this may account for the overall loss of lymphocytes remain to be elucidated. Moreover, Xiong et al. ${ }^{9}$ reported upregulation of apoptosis, autophagy, and p53 pathways in PBMCs of COVID-19 patients. Zheng et al. ${ }^{11}$ suggested functional exhaustion of NK and $\mathrm{CD}^{+}{ }^{+} \mathrm{T}$ cells with increased expression of NKG2A in COVID-19 patients, which could be restored after recovery. These findings indicated that immune disturbance starts early in COVID-19, as a combined result of both direct and bystander effects. Although current observations revealed that these changes might be largely reversible, especially in mild or moderate cases, long-term followup is warranted for further evaluation of the immune function in recovered patients.

We and others have noticed an exceptionally high proportion of aberrant coagulation in severe and critical patients with COVID-19. This was rare for other coronavirus infections, but has been reported in severe influenza. COVID-19 patients exhibited a hypercoagulable state, featured by prolonged prothrombin time, elevated levels of D-dimer and fibrinogen, and near normal activated partial thromboplastin time. A few patients would finally progress to overt disseminated intravascular coagulation (DIC). Tang et al. ${ }^{12}$ reported that $71.4 \%$ of non-survivors and $0.6 \%$ of survivors of COVID-19 showed evidence of overt DIC. Indeed, more patients exhibited latent DIC characterized by a hypercoagulable state, as demonstrated by fibrin thrombus formation in 

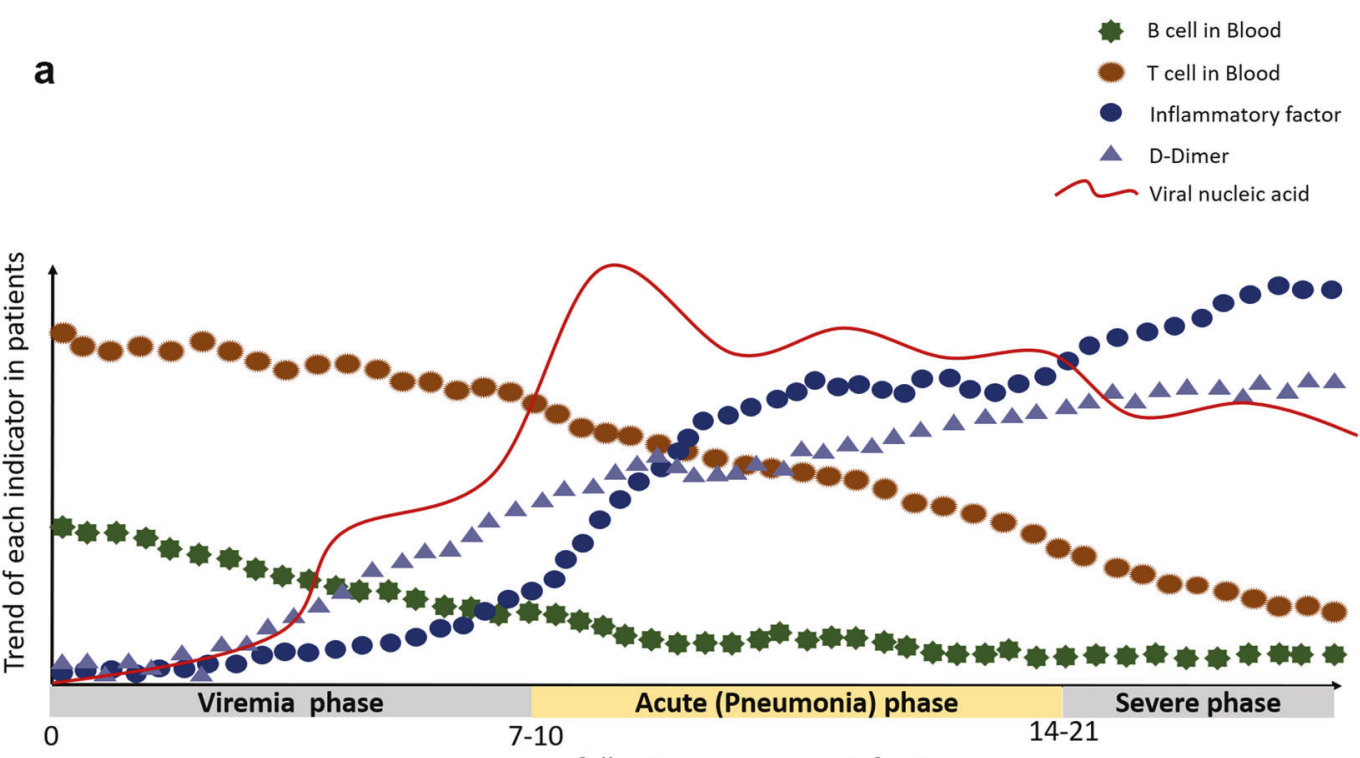

Days following SARS-CoV-2 infection

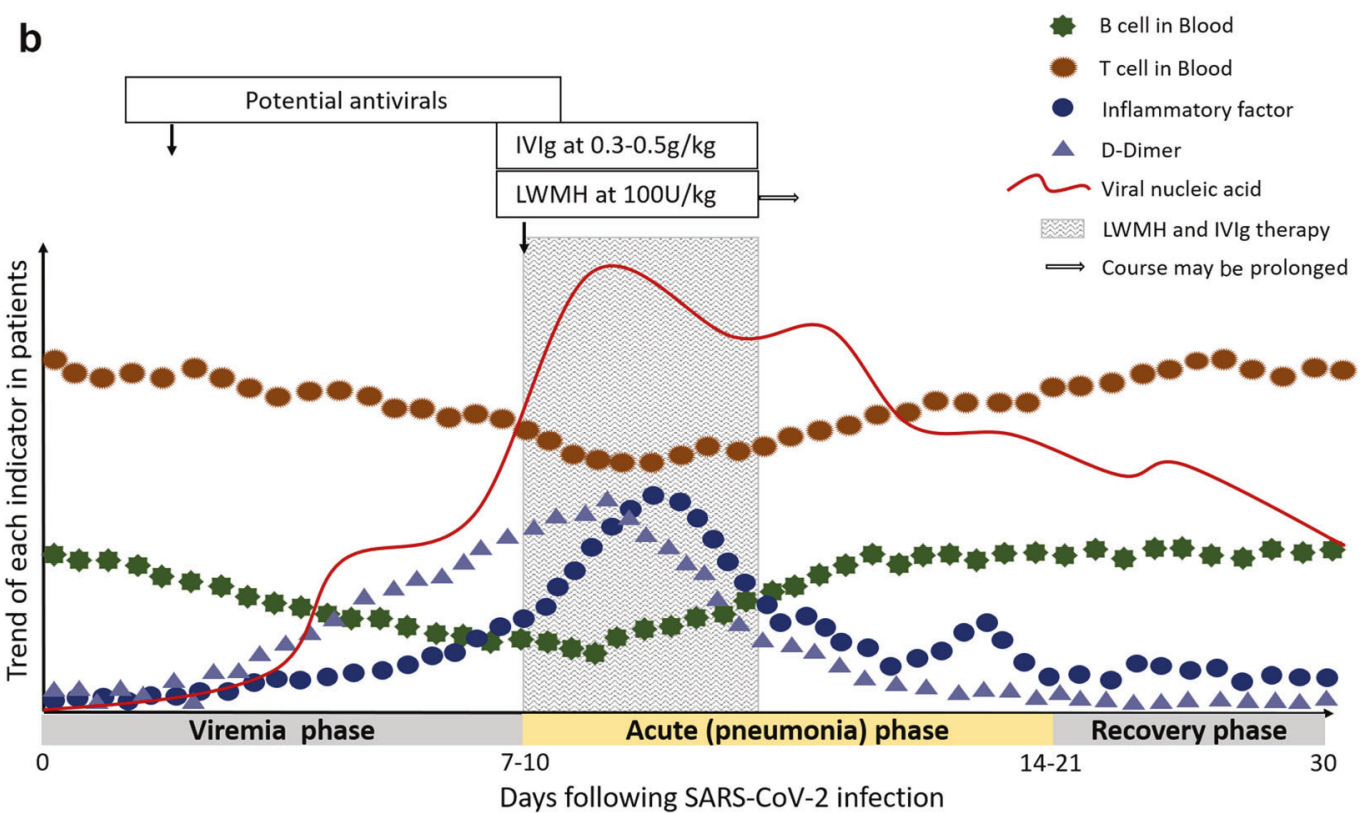

Fig. 1 Hypothetical pathogenesis of COVID-19. The X-axis refers to days after SARS-CoV-2 infection. The disease course is divided into three phases. The $\mathrm{Y}$-axis shows the trend of T cells, B cells, inflammatory factors, D-dimer and viral load in patients. a The trend of each indicator in severe COVID-19 patients. b The trend of each indicator in severe COVID-19 patients after low-molecular-weight heparin (LWMH) and highdose intravenous immunoglobulin (IVIg) therapy. The shaded areas represent the recommended intervention time for LMWH and IVlg treatment.

post-mortem examination. A high proportion of acro-ischemia was also observed in deteriorating patients with COVID-19, indicating a hypercoagulable status before the final onset of overt DIC.

Several factors may contribute to the coagulation disorder in COVID-19 patients. The persistent inflammatory status in severe and critical COVID-19 patients acts as an important trigger for the coagulation cascade. Certain cytokines including IL- 6 could activate the coagulation system and suppress the fibrinolytic system. In the setting of COVID-19, pulmonary and peripheral endothelial injury due to direct viral attack might be an equally important inducer of hypercoagulation. Endothelial cell injury can strongly activate the coagulation system via exposure of tissue factor and other pathways. Moreover, aggressive immune response could also be augmented by dysfunctional coagulation.
These two processes may act in a feed-forward manner towards an uncontrolled endpoint. In addition, the emergence of antiphospholipid antibodies may intensify the coagulopathy. Various levels of anti-cardiolipin and anti- $\beta 2$ GP1 antibodies were detected in our COVID-19 patients, and additional management strategies may be needed. ${ }^{13}$

Based on what we have learned, the clinical course of SARSCoV-2 infection could be divided into three phases: viremia phase, acute phase (pneumonia phase) and severe or recovery phase (Fig. 1). ${ }^{14}$ Patients with competent immune functions and without obvious risk factors (old age, co-morbidities, etc.) may generate effective and adequate immune responses to suppress the virus in the first or second phase without immune over-reaction. In contrast, patients with immune dysfunction may have a higher risk of failing the initial phase and becoming the severe or critical 
type with higher mortality. Therefore, treatment of COVID-19 should be based on the staging of patients, and the window of opportunity may lie between the first and the second phases, when clinical deterioration is observed with evidence of abrupt inflammation and hypercoagulable status. With no proved antivirals, early intervention has mainly focused on the correct timing of disease stages and implementing ways to stop or slow disease progression. Once the patients enter the critical status, no magic bullet could be relied on other than comprehensive management.

There has been no consensus regarding the best management options during patient deterioration. Potential measures include glucocorticoids, high-dose intravenous immunoglobulin, anti-IL$6 \mathrm{R}$ antibody (tocilizumab, etc.), convalescent plasma therapy and other immunomodulators. In our center, given the benefits and risks of each option, high-dose intravenous immunoglobulin treatment at $0.3-0.5 \mathrm{~g}$ per $\mathrm{kg}$ weight per day for five days was recommended to interrupt the inflammatory flare at an early stage. ${ }^{15}$ Meanwhile, we recommend early anticoagulation therapy, preferably low-molecular-weight heparin (LMWH) when D-dimer level is 4 times higher than the upper limit of normal range, unless there is contraindication (Fig. 1b). ${ }^{14}$ Overview of these therapeutic approaches will be discussed by Dr Wenhong Zhang and colleagues in this issue. A take-home message here is that timing is extremely important in this group of patients, regardless of what treatment might be used.

Although management of severe and critical COVID-19 patients is important in reducing the mortality of the ongoing pandemic, the truly key measures lie in prevention, monitoring and timely intervention. To be fully prepared for this ongoing pandemic and any outbreak that might occur in the future, it is crucial to understand the pathogenesis of this disease. We anticipate more studies to facilitate the development of specific therapeutics to control the virus, minimize pulmonary injuries or optimize immune responses.

\section{ADDITIONAL INFORMATION}

Competing interests: The authors declare no competing interests.

\section{REFERENCES}

1. Lu, R. et al. Lancet 395, 565-574 (2020).

2. Wölfel, R. et al. Nature https://doi.org/10.1038/s41586-020-2196-x (2020)

3. Qin, C. et al. Clin. Infect. Dis. https://doi.org/10.1093/cid/ciaa248 (2020).

4. Zhou, F. et al. Lancet 395, 1054-1062 (2020).

5. Xu, Z. et al. Lancet Respir. Med. 8, 420-422 (2020).

6. Li., T. et al. J. Infect. Dis. 189, 648-651 (2004).

7. Gu, J. et al. J. Exp. Med. 202, 415-424 (2005)

8. O'Donnell, R., Tasker, R. C. \& Roe, M. F. BMJ 327, 620 (2003).

9. Xiong, Y. et al. Emerg. Microbes Infect. 9, 761-770 (2020).

10. Wang, X. et al. Cell Mol. Immunol. https://doi.org/10.1038/s41423-020-0424-9 (2020).

11. Zheng, M. et al. Cell Mol. Immunol. https://doi.org/10.1038/s41423-020-0402-2 (2020).

12. Tang, N., Li, D., Wang, X. \& Sun, Z. J. Thromb. Haemost. 18, 844-847 (2020).

13. Zhang, Y. et al. N. Engl. J. Med. https://doi.org/10.1056/NEJMc2007575 (2020).

14. Lin, L., Lu, L., Cao, W. \& Li, T. Emerg. Microbes Infect. 9, 727-732 (2020).

15. Cao, W. et al. Open Forum Infect. Dis. 7, ofaa102 (2020). 